EUROPHYSICS LETTERS

22 May 1997

Europhys. Lett., (), pp. ()

\title{
Charge oscillations in Debye-Hückel theory
}

\author{
Benjamin P. LeE ${ }^{1}$ and Michael E. Fisher ${ }^{2}$ \\ ${ }^{1}$ Polymers Division, National Institute of Standards and Technology, Gaitherburg, Mary- \\ land 20899, USA \\ ${ }^{2}$ Institute for Physical Science and Technology, University of Maryland, College Park, \\ Maryland 20742, USA
}

(received ; accepted )

PACS. 61.20.Qg- Structure of associated liquids: electrolytes, molten salts, etc.

PACS. 05.20.-y- Statistical mechanics.

PACS. 05.70.Jk - Critical point phenomena.

\begin{abstract}
The recent generalized Debye-Hückel (GDH) theory is applied to the calculation of the charge-charge correlation function, $G_{Z Z}(\mathbf{r})$. The resulting expression satisfies both (i) the charge neutrality condition and (ii) the Stillinger-Lovett second-moment condition for all $T$ and $\rho_{N}$, the overall ion density, and (iii) exhibits charge oscillations for densities above a "Kirkwood line" in the $\left(\rho_{N}, T\right)$ plane. This corrects the normally assumed DH charge correlations, and, when combined with the GDH analysis of the density correlations, leaves the GDH theory as the only complete description of ionic correlation functions, as judged by (i)-(iii), (iv) exact low-density $\left(\rho_{N}, T\right)$ variation, and $(\mathrm{v})$ reasonable behavior near criticality.
\end{abstract}

A complete theory of ionic fluids, and in particular one which describes the ionic critical region, remains an outstanding challenge for statistical physics [1]. Recent progress has been made at the mean-field level by studies based on the Debye-Hückel (DH) theory of the restricted primitive model (RPM) of electrolytes [2], supplemented with ion pairing, free-ion depletion, and the concomitant dipolar-ionic interactions [3]. A satisfactory theory must also include a description of the ionic correlation functions. However, the conventionally assumed DH ion-ion correlation functions, of which there appear to be three varieties, have several shortcomings: the most significant is the absence of a diverging density-density correlation length at the DH critical point, which renders the theory totally uninformative as regards the relevant order-parameter fluctuations. Further, although the predicted charge-charge correlation function, $G_{Z Z}(\mathbf{r})$, with the familiar screening decay as $e^{-\kappa_{D} r} / r$, is exact in the low-density limit, it violates the Stillinger-Lovett second-moment condition 清. Furthermore, there is much evidence indicating that the charge correlations become oscillatory at moderately high densities [5, 6], a phenomenon also missed by the simple screening form.

Similar difficulties arise with the oft-used mean-spherical approximation (MSA), which exhibits, in particular, a complete cancellation of Coulombic effects from the density-density

Typeset using EURO-LAT $\mathrm{E}$ X 
correlation function, $G_{N N}(\mathbf{r})$. In this case some improvement has been found by adding an ad hoc term to the assumed direct correlation functions and adjusting it to gain consistency with various sum rules; this generalized MSA, or GMSA, yields non-trivial density correlations, including a diverging correlation length at the MSA critical point [7].

However, it fails badly at low densities [8, 9, 10] and, as explained elsewhere [3, 8, 11], the MSA (and GMSA) appears to be inferior to DH-based theories for describing the critical region. Hence, we have sought to remedy the deficiencies of the DH correlation functions as well, and specifically to do so in a more natural way. By following the spirit of the $\mathrm{DH}$ approximation, we extended the theory to the case of non-uniform, slowly varying ionic densities, $\rho_{ \pm}(\mathbf{r})$, thus enabling derivation of a Helmholtz free energy functional [8, 10]. Ion correlations may then be obtained by functional differentiation techniques. This generalized Debye-Hückel $(\mathrm{GDH})$ theory was applied to the calculation of $G_{N N}(\mathbf{r})$, and provided not only the expected critical divergence of the second-moment density correlation length, $\xi$, but also the surprising, universal and exact divergence of $\xi$ in the low-density limit 8, 12] (where the GMSA fails by predicting a finite, non-universal value [9]). This testament to the physical validity of the GDH approach motivated the calculation of the charge-charge correlations via GDH theory that is reported here.

We find an expression for $G_{Z Z}(\mathbf{r}) \equiv\left\langle\left[\rho_{+}(\mathbf{r})-\rho_{-}(\mathbf{r})\right]\left[\rho_{+}(\mathbf{0})-\rho_{-}(\mathbf{0})\right]\right\rangle$ which in the lowdensity limit approaches the conventional and exact $\mathrm{DH}$ result, but which also explicitly satisfies the Stillinger-Lovett second-moment condition. Furthermore, it exhibits charge oscillations for densities above a "Kirkwood line" in the density-temperature plane [7]. More concretely, we find for the RPM (equisized hard-spheres with diameters $a$, charges $\pm q_{0}$, and solvent dielectric constant $D$ ) the closed-form, Fourier transform expression for the charge-charge correlation function

$$
\hat{G}_{Z Z}\left(\mathbf{k} ; \rho_{N}, T\right)=\rho_{N} k^{2} /\left[\kappa_{D}^{2}+k^{2}+a^{-2} g_{0}\left(\kappa_{D} a, k a\right)\right],
$$

where $\rho_{N}=\rho_{+}+\rho_{-}$is the total ion density while the Debye parameter is given, as usual, by $\kappa_{D}^{2}=4 \pi q_{0}^{2} \rho_{N} / D k_{B} T$, and

$$
g_{0}(x, q)=x^{2}(\cos q-1)-\left[2 \ln (1+x)-2 x+x^{2}\right](\cos q-\sin q / q) .
$$

Expansion in powers of $k$ yields $\hat{G}_{Z Z}\left(\mathbf{k} ; \rho_{N}, T\right)=\left(D k_{B} T / 4 \pi q_{0}^{2}\right) k^{2}+O\left(k^{4}\right)$, which demonstrates satisfaction of both the Stillinger-Lovett second-moment condition,

$$
\int d \mathbf{r} r^{2} G_{Z Z}(\mathbf{r})=-6 \rho_{N} / \kappa_{D}^{2}
$$

and the "zeroth-moment" or charge-neutrality condition,

$$
\int d \mathbf{r} G_{Z Z}(\mathbf{r})=0 \quad \Rightarrow \quad \int_{|\mathbf{r}|>a} d \mathbf{r} G_{Z Z}(\mathbf{r})=-\rho_{N},
$$

for all $\rho_{N}$ and $T$. In the low-density limit (11) becomes $\hat{G}_{Z Z}(\mathbf{k}) \approx \rho_{N} k^{2} /\left(\kappa_{D}^{2}+k^{2}\right)$, the exact, universal limiting behavior. By analyzing the poles of (1i) we may obtain the predicted large-distance behavior of $G_{Z Z}\left(\mathbf{r} ; \rho_{N}, T\right)$ : from that we find that simple exponential decay persists only up to $x \equiv \kappa_{D} a=x_{K}$; for $x>x_{K}$ the decay is oscillatory. Numerically we obtain the "Kirkwood value"

$$
x_{K} \simeq 1.17832,
$$

which lies in the usually expected range 四, 5, 6, 6].

Before presenting the GDH calculation, we summarize briefly the conventional DH correlation functions for comparison. Debye-Hückel theory provides an approximate result for $\phi_{\sigma}(\mathbf{r})$, 
the mean electrostatic potential at $\mathbf{r}$ due to both a charge of type $\sigma$ fixed at the origin and the corresponding induced charge distribution [2], namely,

$$
\begin{aligned}
\phi_{\sigma}^{D H}(\mathbf{r}) & =q_{\sigma} / D r-q_{\sigma} \kappa_{D} / D\left(1+\kappa_{D} a\right), & & r<a, \\
& =q_{\sigma} e^{\kappa_{D}(r-a)} / D\left(1+\kappa_{D} a\right), & & r \geq a .
\end{aligned}
$$

The DH thermodynamics uses only the self-potential $\lim _{\mathbf{r} \rightarrow 0}\left[\phi_{\sigma}^{D H}(\mathbf{r})-q_{\sigma} / D r\right]=q_{\sigma} \kappa_{D} / D(1+$ $\left.\kappa_{D} a\right)$. In a first effort to obtain correlations, one may supplement the treatment of Debye and Hückel with the formally exact statistical Poisson's equation, which for the RPM states [2(b)]

$$
\nabla^{2} \phi_{\sigma}(\mathbf{r})=-\left(4 \pi q_{\sigma} / D\right) G_{Z Z}(\mathbf{r}) / \rho_{N}, \quad(\sigma= \pm) .
$$

This yields a $G_{Z Z}^{\text {Poiss }}$ with a simple screening decay that (i) satisfies (雨) for all $\rho_{N}$ and $T$, but (ii) violates the second-moment condition everywhere except in the low density, $\kappa_{D} a \rightarrow 0$ limit. Furthermore, this Poisson route says nothing whatsoever about the density-density correlations.

A second approach is to parallel the derivation of (6) [2] by putting $\phi_{\sigma}^{D H}(\mathbf{r})$ into a Boltzmann form, $g_{\sigma \tau}(\mathbf{r} \neq \mathbf{0}) \equiv\left\langle\rho_{\sigma}(\mathbf{r}) \rho_{\tau}(\mathbf{0})\right\rangle / \rho_{\sigma} \rho_{\tau} \simeq \exp \left[-\beta q_{\sigma} \phi_{\tau}(\mathbf{r})\right]$ for $r>a$ with $g_{\sigma \tau}(\mathbf{r})=0$ for $r<a$. Then both $G_{Z Z}^{\mathrm{Bltz}}$ and $G_{N N}^{\mathrm{Bltz}}$ may be obtained, although the latter displays no sign of the proper critical behavior. However, a more glaring defect of this approach is that $G_{Z Z}^{\mathrm{Bltz}}(\mathbf{r})$ violates not only the second-moment condition, but also the charge-neutrality sum rule! [This follows readily from the inequality $\sinh x>x$ (when $x>0$ ).]

A third path, perhaps the most travelled in the literature, is to linearize the Boltzmann form to get $g_{\sigma \tau}(\mathbf{r}) \simeq 1-\beta q_{\sigma} \phi_{\tau}(\mathbf{r})$, for $r>a$. This gives the same charge correlations as does the Poisson route, but for $G_{N N}^{\operatorname{lin}}$ the Coulombic terms cancel completely, as in the MSA.

Now, although (7) is an exact relation, approximate theories are generally inconsistent with respect to some identities; indeed, only the exact solution can satisfy all possible relations. Our GDH theory [8] provides an alternate, formally exact approach to the correlations, which more closely follows the thermodynamic theory and may then be judged on its relative merits.

Our guiding motivation is that a free-energy-functional formulation ensures density correlations that are sensitive to the critical point (i.e., the compressibility relation is satisfied by construction); thus, we are led to develop a $\mathrm{DH}$ theory for an inhomogeneous electrolyte. As detailed elsewhere [10], the approach is sufficiently general to allow the calculation, via functional differentiation techniques, of all the $\sigma, \tau$ correlation functions $G_{\sigma \tau}(\mathbf{r})$ for an arbitrary multi-component, equisized hard-sphere electrolyte. For brevity, however, we restrict consideration here to the RPM, for which the imposed density variations

$$
\rho_{ \pm}(\mathbf{r})=\bar{\rho}_{ \pm}[1 \pm \Delta \cos \mathbf{k} \cdot \mathbf{r}],
$$

(i.e., in response to an external potential) result in the Helmholtz free energy

$$
\bar{f}\left[\rho_{\sigma}(\mathbf{r})\right]-\bar{f}\left[\bar{\rho}_{\sigma}\right]=-\frac{1}{4} \rho_{N}^{2} \hat{G}_{Z Z}^{-1}(\mathbf{k}) \Delta^{2}+O\left(\Delta^{4}\right),
$$

where $\bar{f} \equiv-\beta F / V[8,10]$. Thus $G_{Z Z}$ may be found by expansion in $\Delta$.

The generalized Debye charging process yields the free energy [10, 13

$$
F\left[\rho_{\sigma}(\mathbf{r})\right]=F^{H C}\left[\rho_{\sigma}(\mathbf{r})\right]+\int_{0}^{1} d \lambda \sum_{\sigma} \int d \mathbf{r}^{\prime} \rho_{\sigma}\left(\mathbf{r}^{\prime}\right) \psi_{\sigma}\left(\mathbf{r}^{\prime},\left\{\lambda q_{\sigma}\right\}\right) .
$$

where $F^{H C}$ denotes the pure hard-core Helmholtz free energy functional while the mean electrostatic potential seen by an ion of type $\sigma$ fixed at $\mathbf{r}^{\prime}$ is found from

$$
\psi_{\sigma}\left(\mathbf{r}^{\prime}\right) \equiv \lim _{\mathbf{r} \rightarrow \mathbf{r}^{\prime}}\left[\phi_{\sigma}\left(\mathbf{r} ; \mathbf{r}^{\prime}\right)-q_{\sigma} / D\left|\mathbf{r}-\mathbf{r}^{\prime}\right|\right],
$$


in which $\phi_{\sigma}\left(\mathbf{r} ; \mathbf{r}^{\prime}\right)$ is the potential at $\mathbf{r}$ due to both the fixed charge at $\mathbf{r}^{\prime}$ and the induced charge distribution [8, 10]. [Compare with (6) above, et seq.] To calculate $\phi_{\sigma}\left(\mathbf{r} ; \mathbf{r}^{\prime}\right)$ we begin with the exact inhomogeneous version of the statistical Poisson's equation [10],

$$
\nabla_{r}^{2} \phi_{\sigma}\left(\mathbf{r} ; \mathbf{r}^{\prime}\right)=-(4 \pi / D) \sum_{\tau} q_{\tau} \rho_{\tau}(\mathbf{r}) g_{\tau \sigma}\left(\mathbf{r} ; \mathbf{r}^{\prime}\right) .
$$

The DH approximation is to replace the $g_{\tau \sigma}$ with Boltzmann factors which depend on the potential. However, it is crucial to note that the varying ionic charge densities carry along an imposed overall electrostatic potential $\Phi(\mathbf{r})$, determined simply by

$$
\nabla^{2} \Phi(\mathbf{r})=-4 \pi q_{0} \rho_{Z}(\mathbf{r}) / D \equiv-(4 \pi / D) \sum_{\sigma} q_{\tau} \rho_{\tau}(\mathbf{r}),
$$

with appropriate boundary conditions. This is independent of the fixed charge of type $\sigma$ at $\mathbf{r}^{\prime}$, and therefore should not contribute to the Boltzmann factor for $g_{\tau \sigma}$. Hence, in the spirit of $\mathrm{DH}$, we take

$$
g_{\tau \sigma}\left(\mathbf{r} ; \mathbf{r}^{\prime}\right) \simeq \exp \left[-\beta q_{\tau} \widetilde{\phi}_{\sigma}\left(\mathbf{r} ; \mathbf{r}^{\prime}\right)\right], \quad\left|\mathbf{r}-\mathbf{r}^{\prime}\right|>a,
$$

with the "local induced potential"

$$
\widetilde{\phi}_{\sigma}\left(\mathbf{r} ; \mathbf{r}^{\prime}\right) \equiv \phi_{\sigma}\left(\mathbf{r} ; \mathbf{r}^{\prime}\right)-\Phi(\mathbf{r}),
$$

and, as before, $g_{\tau \sigma}=0$ for $\left|\mathbf{r}-\mathbf{r}^{\prime}\right|<a$. The need for the separation of $\phi_{\sigma}\left(\mathbf{r} ; \mathbf{r}^{\prime}\right)$ into a background $\Phi(\mathbf{r})$ and an induced piece $\widetilde{\phi}_{\sigma}\left(\mathbf{r} ; \mathbf{r}^{\prime}\right)$ is clear in the limit $\left|\mathbf{r}-\mathbf{r}^{\prime}\right| \rightarrow \infty$, in which $\ln \left[g_{\tau \sigma}\left(\mathbf{r} ; \mathbf{r}^{\prime}\right)\right]$ must vanish while $\phi_{\sigma}\left(\mathbf{r} ; \mathbf{r}^{\prime}\right) \rightarrow \Phi(\mathbf{r})[14]$.

Next one inserts (14) into (12) and makes the second approximation of the DH procedure, namely linearization. This results in the full GDH equation

$$
\begin{aligned}
\nabla_{r}^{2} \widetilde{\phi}_{\sigma}\left(\mathbf{r} ; \mathbf{r}^{\prime}\right) & =-(4 \pi / D)\left[q_{\sigma} \delta\left(\mathbf{r}-\mathbf{r}^{\prime}\right)-q_{0} \rho_{Z}(\mathbf{r})\right], & & \left|\mathbf{r}-\mathbf{r}^{\prime}\right| \leq a, \\
& =\tilde{\kappa}_{D}^{2}(\mathbf{r}) \widetilde{\phi}_{\sigma}\left(\mathbf{r} ; \mathbf{r}^{\prime}\right), & & \left|\mathbf{r}-\mathbf{r}^{\prime}\right| \geq a,
\end{aligned}
$$

where the spatially varying Debye parameter [6, ] is defined by $\tilde{\kappa}_{D}^{2}(\mathbf{r}) \equiv(4 \pi / D) \sum_{\tau} q_{\tau}^{2} \rho_{\tau}(\mathbf{r})$. The second term on the righthand side for $\left|\mathbf{r}-\mathbf{r}^{\prime}\right| \leq a$, i.e., inside the hard sphere, is needed to cancel the background charge density $\rho_{Z}(\mathbf{r})$ there; it represents an effective "cavity source" term.

To obtain $G_{Z Z}$ for the RPM we chose the spatially varying densities (8), for which $\tilde{\kappa}_{D}^{2}(\mathbf{r})$ reduces simply to $\kappa_{D}^{2}$. The resulting GDH equation (16) can be solved readily by Green's function methods [8, 10]. Integrating the self-potential $\psi_{\sigma}\left(\mathbf{r}^{\prime}\right)$ against the density and charging according to (10) gives the free energy to order $\Delta^{2}$, from which our main result (11) follows by use of (9). Note that the $q_{\sigma} \delta\left(\mathbf{r}-\mathbf{r}^{\prime}\right)$ source does not contribute directly to the charge-charge correlation function in the simple case of the RPM; rather it is the cavity term and $\Phi(\mathbf{r})$ that serve to determine $G_{Z Z}$.

To elucidate the long distance behavior of $G_{Z Z}(\mathbf{r})$ we solve for the pole, $k_{0}$, of $\hat{G}_{Z Z}(\mathbf{k})$ that lies closest to the origin in the complex $k$ plane. The real and imaginary parts of $k_{0}$ plotted in fig. 1 were found by solving the coupled equations $\operatorname{Re}\left[\hat{G}_{Z Z}^{-1}\left(k_{0}\right)\right]=\operatorname{Im}\left[\hat{G}_{Z Z}^{-1}\left(k_{0}\right)\right]=$ 0 numerically, using the Newton-Raphson method. When this pole is purely imaginary, corresponding to the leftmost part of curve (a) in fig. 1, $G_{Z Z}$ decays monotonically as $e^{-r / \xi_{z}} / r$, where the screening length is $\xi_{Z}=1 / \operatorname{Im}\left(k_{0}\right)$. In the low-density limit one finds $\xi_{Z}^{-1}=$ $\kappa_{D}\left[1+\frac{1}{4} x^{2}-\frac{1}{9} x^{3}+\frac{19}{96} x^{4}+\ldots\right]$ so that $\xi_{Z}$ approaches the Debye value $\xi_{D} \equiv 1 / \kappa_{D}$ : see curve (c). As $\kappa_{D}$ and $\rho_{N} / T$ increase, the pole $k_{0}$ and a nearby, purely imaginary pole $k_{1}$, curve (d), approach: at the Kirkwood value, $\kappa_{D} a=x_{K}$ [see (5)], they merge, with 


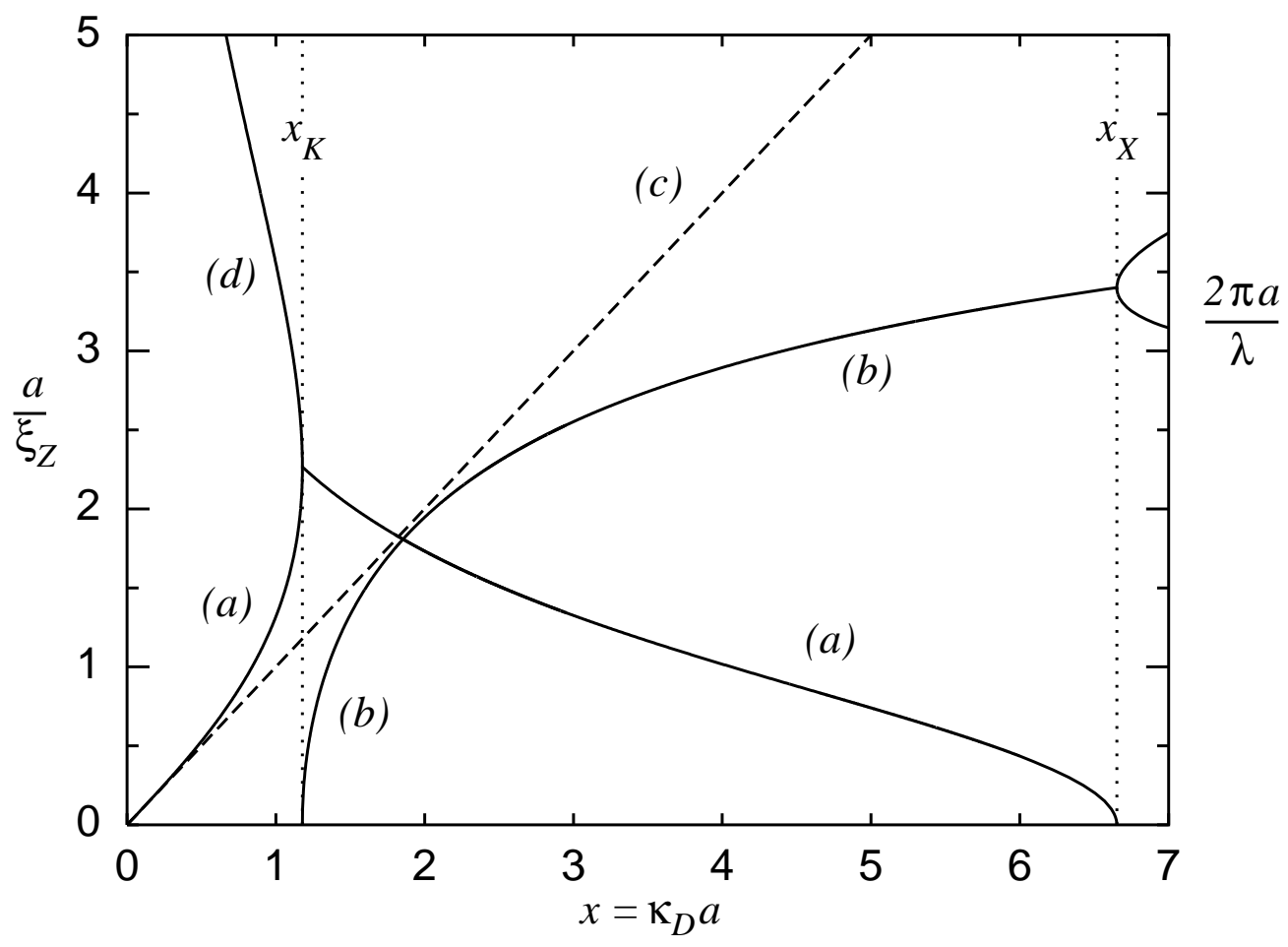

Fig. 1. - Plots versus $x=\left(4 \pi q_{0}^{2} \rho_{N} a^{2} / D k_{B} T\right)^{1 / 2}$ of (a) $\operatorname{Im}\left(k_{0} a\right)=a / \xi_{Z}$, the inverse chargecharge correlation length, (b) $\operatorname{Re}\left(k_{0} a\right)=2 \pi a / \lambda$, the inverse charge oscillation wavelength, (c) $x=\kappa_{D} a$, the inverse Debye length, and (d) $\operatorname{Im}\left(k_{1} a\right)$, the subleading pole. Note that within pure DH theory the critical point occurs at $x_{c}=1$ and $T_{c}^{*}=1 / 16$; in pairing theories based on DH theory $x_{c}=0.9-1.1$ and $T_{c}^{*}=0.052-0.057[1,3,15]$.

$a / \xi_{Z}=2.266_{0}$, and for larger $\kappa_{D}$ they become complex, implying the oscillatory decay $G_{Z Z}(\mathbf{r}) \sim \cos \left[(2 \pi r / \lambda)-\theta_{\kappa_{D}}\right] e^{-r / \xi_{Z}} / r$, with $\lambda=2 \pi / \operatorname{Re}\left(k_{0}\right)$ [see plot (b)] and $\theta_{\kappa_{D}}$ a phase shift. Hence, charge oscillations occur for densities $\rho_{N}^{*}=\rho_{N} a^{3}>x_{K}^{2} T^{*} / 4 \pi \simeq 0.110 T^{*}$, where $T^{*} \equiv D k_{B} T a / q_{0}^{2}$. Finally, at $x=x_{X}=6.6523_{2}$ the poles move to the real axis, i.e., they merge with their complex conjugates. Here the oscillations, with wavelength $\lambda_{X} \simeq 1.847 a$, are no longer damped; this is suggestive of the onset of crystallization [6] although this region certainly lies beyond the limits of validity of our approximation.

As a consequence of our analysis one sees that the GDH theory for ion correlations is the only one available that satisfies the requisite sum rules (3) and (4), gives exact results for $G_{Z Z}(\mathbf{r})$ and $G_{N N}(\mathbf{r})$ in the low density limit [8, 12], and behaves sensibly in the critical region, predicting (mean-field-like) diverging density fluctuations [8].

Finally, we remark on the addition of dipolar ion pairing. Following [3] and [8], one may straightforwardly add pairing to the calculation of $G_{Z Z}$. However, in this case, as opposed to the density-density correlations, one expects there to be no major contribution from the pairs, since, in the center-of-mass approximation, the dipolar ion pairs, of density $\rho_{2}$, appear as neutral objects that cannot contribute directly to the charge-charge correlations. One important role of pairing, however, concerns the location of the Kirkwood line in the $\left(\rho_{N}, T\right)$ plane: owing to the depletion of the free ion density, $\rho_{1} \equiv \rho_{+}+\rho_{-}=\rho_{N}-2 \rho_{2}$, the onset 
of charge oscillations is expected to lie close to the critical region: see 11, 15 for the critical parameters and degree of pairing in the various approximations. Lastly, we mention that the charge-charge correlations in the GDH formulation with pairing still satisfy the sum rules (3) and (4); however, in the Stillinger-Lovett rule (3) the "background" dielectric constant $D$ that enters the definition of $\kappa_{D}$ acquires a linear dependence on $\rho_{2}\left(\rho_{N}, T\right)$. In general, the state-dependence of $D$ seems an open question [ [1] although most authors seem to hold that it should be totally absent in the RPM. If that is correct the pairing treatment would require further improvement. (See also [11.)

The insight underlying this work arose from discussions with Professor Jean Pierre Hansen and from his lectures and lecture notes which are gratefully acknowledged. The interest of Dr. Jack Douglas, Dr. Stefan Bekiranov and Daniel M. Zuckerman has been appreciated. Our researches have been supported by NSF Grant Nos. CHE 93-11729 and 96-14495 and by a National Research Council Research Associateship.

\section{REFERENCES}

[1] See, e.g., Fisher M.E., J. Stat. Phys., 75 (1994) 1; ibid. J. Phys. Condens. Matt., 8 (1996) 1.

[2] (a) Debye P. and Hückel E., Phys. Z., 24 (1923) 185. For a modern presentation, and the low-density results, see (b) D. A. MCQUARRIE, Statistical Mechanics, (Harper-Collins, NY, 1976), chap. 15.

[3] (a) Fisher M.E. and Levin Y., Phys. Rev. Lett., 71 (1993) 3826, (b) Levin Y. and Fisher M.E., Physica A, 225 (1996) 164.

[4] (a) Stillinger F.H. and Lovett R., J. Chem. Phys., 48 (1968) 3858, (b) ibid. J. Chem. Phys., 49 (1968) 1991.

[5] Kirkwood J.G., Chem. Rev., 19 (1936) 275, found $x_{K} \simeq 1.03$.

[6] Outhwaite C.W., in Statistical Mechanics, A Specialist Periodical Report, edited by K. Singer, Vol. 2 (London: The Chemical Society) pp. 188; the linearized modified Poisson-Boltzmann (MPB) theory yields $x_{K} \simeq 1.241$.

[7] Leote de Carvalho R.J.F. and Evans R., (a) Molec. Phys., 83 (1994) 619, (b) J. Phys. Condens. Matter, 7 (1995) L575; analysis of the GMSA gives $x_{K} \simeq 1.228$.

[8] LeE B.P. and Fisher M.E., Phys. Rev. Lett., 76 (1996) 2906.

[9] In 8 replace the first part of equation $(13)$ by $\xi_{\mathrm{GMSA}} \approx\left(\frac{1}{8} a b\right)^{1 / 2}$ and modify the following sentence by replacing $\xi_{\mathrm{GMSA}}$ with $\xi_{\infty \mathrm{GMSA}}$.

[10] LEE B.P. and Fisher M.E., [to be published].

[11] Zuckerman D.M., Fisher M.E. and Lee B.P., [submitted for publication].

[12] Bekiranov S. and Fisher M.E., [in preparation] based on the analysis of Meeron E., J. Chem. Phys., 28 (1958) 630.

[13] Note that all but the ideal gas components of $F^{H C}$ cancel out of (9).

[14] For locally charge-neutral density variations, as considered in $[\mathbb{8}], \Phi(\mathbf{r})$ vanishes and the separation (15) is unnecessary.

[15] Fisher M.E. and Lee B.P., Phys. Rev. Lett., 77 (1996) 3561. 